\title{
Multi-band modelling of appearance
}

\author{
M.B. Stegmann*, R. Larsen \\ Informatics and Mathematical Modelling, Technical University of Denmark, DTU, Richard Petersens Plads, Building 321, DK-2800 Kgs. Lyngby, Denmark
}

\begin{abstract}
Earlier work has demonstrated generative models capable of synthesising near photo-realistic grey-scale images of objects. These models have been augmented with colour information, and recently with edge information. This paper extends the active appearance model framework by modelling the appearance of both derived feature bands and an intensity band. As a special case of feature-band augmented appearance modelling we propose a dedicated representation with applications to face segmentation. The representation addresses a major problem within face recognition by lowering the sensitivity to lighting conditions. Results show that the localisation accuracy of facial features is considerably increased using this appearance representation under diffuse and directional lighting and at multiple scales.

(C) 2002 Elsevier Science B.V. All rights reserved.
\end{abstract}

Keywords: Generative model; Active appearance model; Lighting invariance; Face recognition; Segmentation

\section{Introduction}

Models capable of synthesising complete images of objects have over the past few years proven their worth when interpreting images. Applications include real-time tracking of deformable objects [1,2], face recognition [3-5], and recovery of anatomical structures in magnetic resonance images [6-9], ultrasound images [10] and X-rays $[9,11]$. The key idea in all of these generative models is to perform a per-pixel comparison between unseen input images and synthesised images and subsequently drive these to equality.

In this paper, we investigate a generative model that has proven widely applicable. The active appearance models (AAMs) [12,13] have been applied to most of the examples given above. As Cootes and Taylor [14] we model the appearance of edge strength, but in contrast this is also augmented with colour information and conventional raw intensities. We show that a considerable gain in accuracy can be achieved, merely by selecting a more appropriate representation of the particular object class being modelled. This paper demonstrates that mature image processing methods can co-exist in rewarding symbiosis with a modern generative model-based vision technique.

\section{Active appearance models}

Active appearance models (AAMs) [12,13] establish a compact parameterisation of object variability, as learned from a training set by estimating a set of latent variables. The modelled object properties are usually shape and pixel intensities. The latter is henceforward denoted texture. From these quantities new images similar to the training set can be generated.

Objects are defined by marking up each example with points of correspondence over the set either by hand, or by semi- to completely automated methods. The key to the compactness of these models is a proper compensation of shape variability prior to modelling texture variability. Models omitting this shape compensation, such as the Eigen-face model [15], experience major difficulties in modelling texture variability in a compact manner.

By exploiting prior knowledge about the local nature of the optimisation space, these models can be fitted to unseen images in a fraction of a second, given a reasonable initialisation.

Variability is modelled by means of a principal component analysis (PCA), i.e. an eigen analysis of the dispersions of shape and texture. Shapes are brought into alignment using a generalised procrustes analysis (GPA) [16], and textures are warped into correspondence using a thin-plate spline [17] or a piece-wise affine warp, thereby compensating for any variation in shape. Let $\overline{\mathbf{x}}$ and $\overline{\mathbf{t}}$ denote the shape and texture mean, respectively. The (ranked) model parameters, c, can then generate new instances in

\footnotetext{
* Corresponding author.

E-mail address: mbs@imm.dtu.dk (M.B. Stegmann).
} 
a simple linear manner

$\mathbf{x}=\overline{\mathbf{x}}+\boldsymbol{\Phi}_{s} \mathbf{c}, \quad \mathbf{t}=\overline{\mathbf{t}}+\boldsymbol{\Phi}_{t} \mathbf{c}$,

where $\boldsymbol{\Phi}_{s}$ and $\boldsymbol{\Phi}_{t}$ are eigenvectors obtained from the training set. The object instance, $(\mathbf{x}, \mathbf{t})$, is synthesised into an image by warping the pixel intensities of $\mathbf{t}$ into the geometry of the shape $\mathbf{x}$.

By defining a suitable measure of fit, $M(\mathbf{c}, \mathbf{I})$, the model could be matched to an unseen image, I, using standard optimisation techniques such as conjugate-gradient, Levenberg-Marquardt or Metropolis-Hastings in a simulated annealing scheme. However, AAMs take a different approach where residual vectors between the model and image, $\delta \mathbf{t}=\mathbf{t}_{\text {model }}-\mathbf{t}_{\text {image }}$ are regressed against known displacement vectors, $\delta \mathbf{c}$, using principal components regression:

$\delta \mathbf{c}=\mathbf{R} \delta \mathbf{t}$.

Embedded into an iterative updating scheme, this has proven to be a very efficient way of matching these models to unseen images. For large models (many texture samples) built on large training sets, this approach becomes quite resource demanding w.r.t. memory and computation. However, recent experiments [18] have shown that estimating the Jacobian, $\partial(\delta \mathbf{t}) / \partial \mathbf{c}$, over the training set using a simple weighting scheme, in practice yields better results than the regression approach with far less computational and memory requirements. In the work below the regression approach has been taken.

This sums up the basic theory of AAMs. For further details refer to Refs. [13,18,19].

\section{Multi-band AAMs}

Contrary to the above univariate view of images, the most frequently used image source - the RGB camera-is multivariate. Thus, collapsing the red, green and blue band into a single intensity band looses specificity. As Edwards et al. [12] we model multiple texture bands by simple concatenation. Any correlation between bands is to be picked up by the PCA analogue to the recovered correlation along shape contours. The concept of texture is consequently extended to encapsulate any corresponding measurement over the training set. Let $m$ denote the number of texture samples in band $i$ :

$\mathbf{u}_{i}=\left[\begin{array}{llll}u_{i 1} & u_{i 2} & \cdots & u_{i m}\end{array}\right]$.

The concatenated texture vector will then be for $p$ texture bands:

$\mathbf{t}=\left[\begin{array}{llll}\mathbf{u}_{1} & \mathbf{u}_{2} & \cdots & \mathbf{u}_{p}\end{array}\right]$.

Henceforth all AAM processing is left unchanged. This is multi-band modelling of appearance. As hinted this approach can be taken to all structures of corresponding input data, three dimensional problems, time-series [7],
$3 \mathrm{D}+$ time, etc. Often, the hard part is to obtain the correspondence, in particular for cases with sparse or incomplete data.

\section{The VHE representation}

As a special case of multi-band appearance we propose a representation suitable for segmentation of face-like images. A secondary aim is to stress the ease with which one can add feature bands to create new representations suitable for a particular domain.

The statistical approach to model building has many striking advantages. Variability, dependencies, etc. are learned (estimated) from representative example solutions contrary to being designed (coded) explicitly into the model. However, some sources of variation are harder to generalise than others. Given a few people who smile it is a reasonable task to build a complete model of smiling mouths, i.e. a model that generalise well. This is due to the low intrinsic dimensionality of the geometrical deformation involved in a smile. On the contrary, lighting effects on a face are very hard to describe. The intrinsic dimensionality is high; 3D geometry of the face, skin surface (dry, sweaty), lighting (type, position, colour), etc.

As an alternative to learning the effects of lighting such as shadows and highlights, we propose a representation less sensitive to these. First, we notice that lighting effects have less influence on the hue band in the hue, saturation and value (HSV) colour space. By modelling hue, we aim at obtaining the specificity of colour models without the sensitivity to effects of lighting. Second, as [14] we notice that edge estimators per se are less sensitive to lighting effects than raw intensities. Since edge estimators are implemented as numeric differential operators (e.g. Sobelfilters) these are unfortunately inherently sensitive to noise, which calls out for some degree of regularisation. This is often achieved through a modest filtering with a Gaussian kernel (preferably of the differential operator). Since this damps the high frequency content of an image, which is less desirable in a segmentation application, we choose to retain a pure intensity-based band. All together these three bands form the value, hue and edge (VHE) representation:

- V, value-the value (intensity) in the HSV colourspace.

- H, modified hue-the angular hue, $h$, of an HSV representation. Here modified to accommodate singleband storage. Since faces have little hue variation, the hue circle is here collapsed around the approximate circular mean, $\theta=0$ and $\theta+\pi$ in the following way:

$h_{\text {mod }}=\left\{\begin{array}{ll}h & \text { if } h<\pi \\ 2 \pi-h & \text { otherwise }\end{array}\right.$. 
Though this introduces ambiguity in hues we expect this to be acceptable compared to the effects of wrapping angles.

- E, edge - the edge strength, calculated as the gradient magnitude

$g=\sqrt{g_{x}^{2}+g_{y}^{2}}$,

where $g_{x}$ and $g_{y}$ are horizontal and vertical gradient images obtained from numeric differential operators with a suitable amount of Gaussian smoothing.

\section{Implementation}

All conducted experiments were based on an extended version of the AAM-API, which is an AAM implementation in $\mathrm{C}++$ by one of the authors. A beta version of the AAM-API can be downloaded from http://www.imm.dtu. $\mathrm{dk} / \sim$ aam/. This page also gives several examples on AAMs in other contexts.

\section{Experiments}

To test the hypotheses regarding the described VHE representation a database of 74 face images was compiled:

- Set A-37 people facing front to the camera with a neutral facial expression. Lighting conditions were neutral using diffuse light from above.

- Set B-the same 37 people facing front to the camera with a (new) neutral facial expression. A directional light source was added to simulate partial non-diffuse lighting conditions, i.e. horizontal lighting from the right, as seen from the camera.

Still images were acquired using a Sony DV video camera (DCR-TRV900E PAL) in $640 \times 480$ JPEG colour format and subsequently annotated using 58 landmarks. Refer to Fig. 1 for example images from Set A and B.

Grey-scale versions were obtained using the luminance RGB-weighting scheme:

$G=0.30 R+0.59 G+0.11 B$.

Alternatively, a principal component or maximum autocorrelation factor transform [20] could be applied to the RGB bands to obtain grey-scale versions.

VHE versions of Set A and B were obtained using the procedure described previously. Refer to Fig. 2 for an example VHE transform. Notice the markedly lower horizontal resolution in the modified hue band. This is due to the internal subsampling of the chrominance bands in the video formation and the JPEG compression scheme.

In all experiments AAMs were initialised using a sparse global search exploiting the convergence distance of each
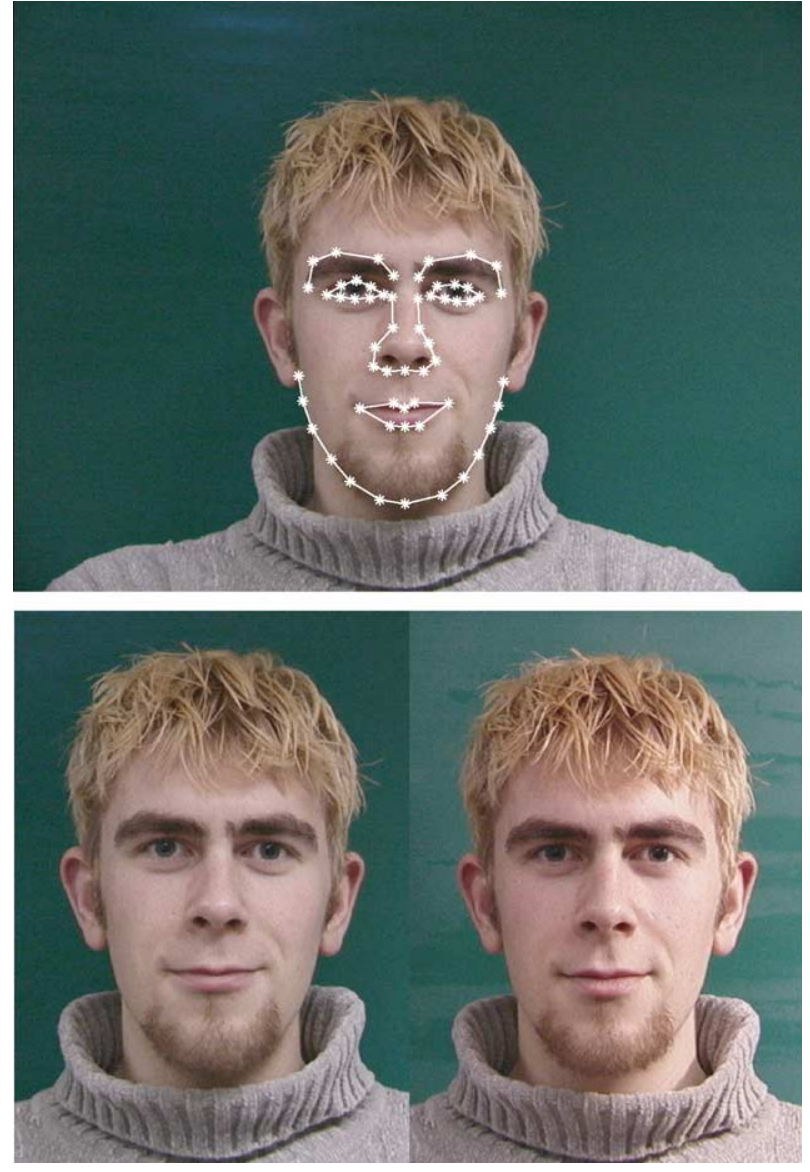

Fig. 1. Top row: example annotation. Bottom row: cropped example images from Set A using diffuse lighting (left) and Set B using directional lighting (right).

parameter. Often this is only done in a few selected parameters. In this case position and scale were adequate. From the results of the global search a candidate set is chosen and iterated further until convergence. The best of these converged results denotes the initial position. To improve speed and robustness this is done on models built at multiple scales. For details see Ref. [21].

\subsection{Segmentation of unknown identity using diffuse lighting}

To assess the segmentation capabilities under standardised lighting conditions cross-validation were carried out on three different AAM representations of Set A: grey-scale, colour and VHE. To obtain optimal performance a leaveone-out scheme was used. Thus, 37 models were built from 36 examples each leading to 37 evaluations of each representation. Input images were subsampled to $320 \times 240$ pixels prior to any AAM processing. The texture models were $\sim 8000$ pixels/band and it took on average 28 combined parameters to represent $95 \%$ of the variation observed in the training set.

The results in Table 1 show a subtle increase in accuracy for the VHE representation compared to the standard greyscale AAM. 

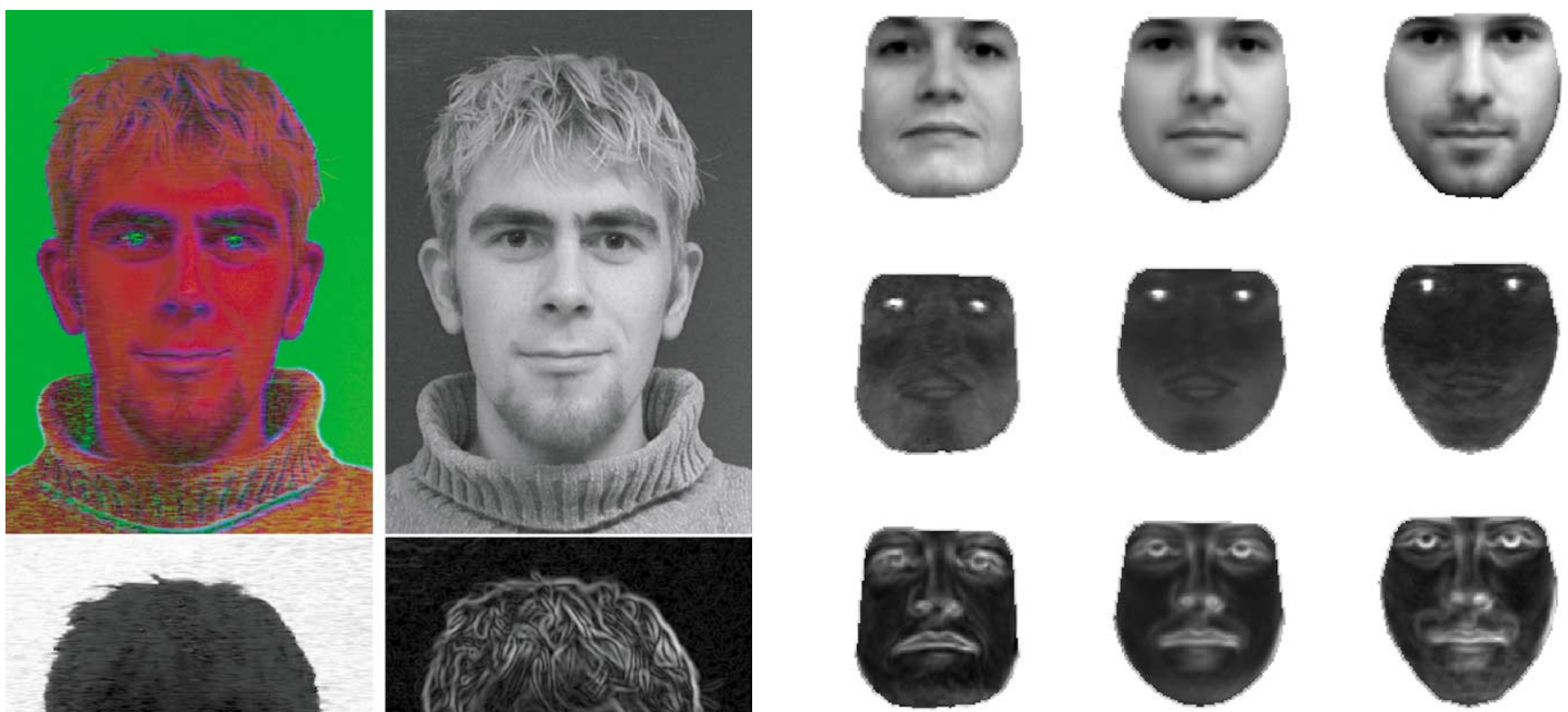

Fig. 3. First combined principal mode, $c_{1}$, for a VHE AAM built on Set A. Value, modified hue and edge bands are shown row-wise, top-down. The deformations are $c_{1}=-3 \sigma_{1}$ (left), mean (middle) and $c_{1}=3 \sigma_{1}$ (right) where $\sigma_{1}$ is one standard deviation over the training set. Bands are stretched linearly for display.

\subsection{Segmentation of known identity using directional lighting}

Subsequently, the three representations were tested for their ability to segment known faces with subtle changes in expression but major changes in lighting. This was carried out by building three AAMs in grey-scale, colour and VHE, respectively, on Set A $(320 \times 240$ pixels). Refer to Fig. 3 for the first principal mode of the VHE AAM.

All three models were subsequently tested on all images in Set $\mathrm{B}$. Table 2 shows an increase in segmentation accuracy of $17 \%$ (pt. - pt.) and $21 \%$ (pt. - crv.) for VHE compared to greyscale. Further, the VHE has lower uncertainty estimates. From the error distributions in Fig. 4 it is noted that the VHE has a lower maximum error and in general a tail less heavy than the grey-scale and the colour $\mathrm{AAM}^{1}$.

\subsection{Accuracy at different scales}

Occasionally, it is not feasible to build AAMs in the original input resolution. This can be due to constraints such as memory consumption, computation time, etc. In a case with high-resolution input but a constraint on the model size one could ask whether a multi-band model should be chosen over a single-band model with a higher resolution.

To test this 18 AAMs were built in six different resolutions using each of the three representations, greyscale, colour and VHE. From Set A 27 examples were selected for training. Image resolutions spanned from

\footnotetext{
${ }^{1}$ The break-ups in the log-plot curves for the grey-scale and colour AAM are due to histogram bins with zero entries.

Leave-one-out segmentation results

\begin{tabular}{lll}
\hline & Mean pt. - pt. & Mean pt. crv. \\
\hline Grey-scale & $2.73 \pm 0.78$ & $1.35 \pm 0.46$ \\
Colour & $2.84 \pm 0.75$ & $1.35 \pm 0.40$ \\
VHE & $2.63 \pm 0.64$ & $1.27 \pm 0.40$ \\
\hline
\end{tabular}


Table 2

Segmentation results using directional lighting

\begin{tabular}{lll}
\hline & Mean pt. - pt. & Mean pt. - crv. \\
\hline Grey-scale & $3.51 \pm 0.85$ & $1.78 \pm 0.49$ \\
Colour & $3.22 \pm 0.67$ & $1.67 \pm 0.44$ \\
VHE & $2.91 \pm 0.65$ & $1.40 \pm 0.36$
\end{tabular}

$108 \times 80$ pixels to full input resolution at $640 \times 480$ pixels. The resulting model sizes were in the range 850-92,118 texture samples. Using the described initialisation method all 18 models were evaluated against the remaining 10 examples of Set A. In Fig. 5 the mean pt.-pt. error is plotted against the model size. Here, pt.-pt. errors are measured in units of pixel width at the used resolution. While the VHE performs best, Fig. 5 stresses the fact that a simple pt.-pt. measure is worthless as performance indicator without the image resolution or model size given.

In a typical benchmarking scheme pixel distances relates to a physical measure. In this experiment we define the measure a $P W I$ which is the Pixel Width at the original Input resolution, i.e. $640 \times 480$ pixels. The PWI equivalent of Fig. 5 is shown in Fig. 6. From the zoom in Fig. 6 (bottom) it
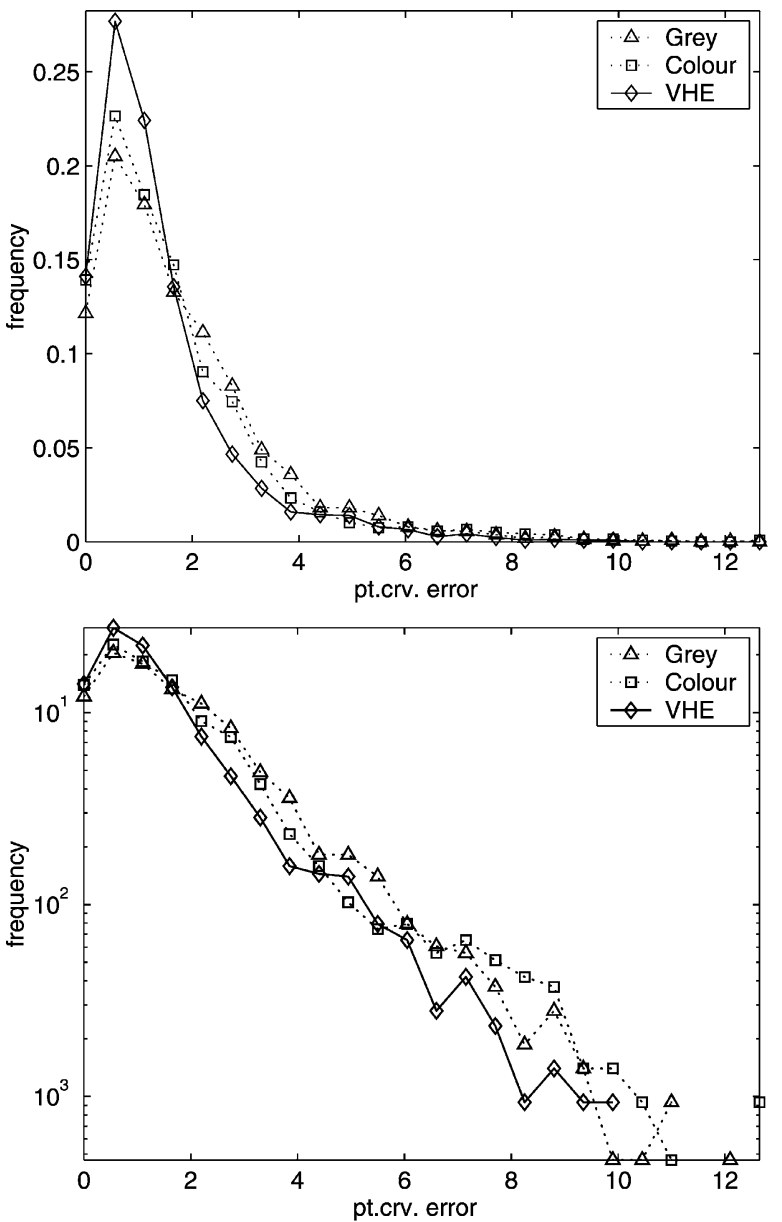

Fig. 4. Distribution of pt.-crv. errors using directional lighting. Shown as normal (top) and log (bottom) plots.
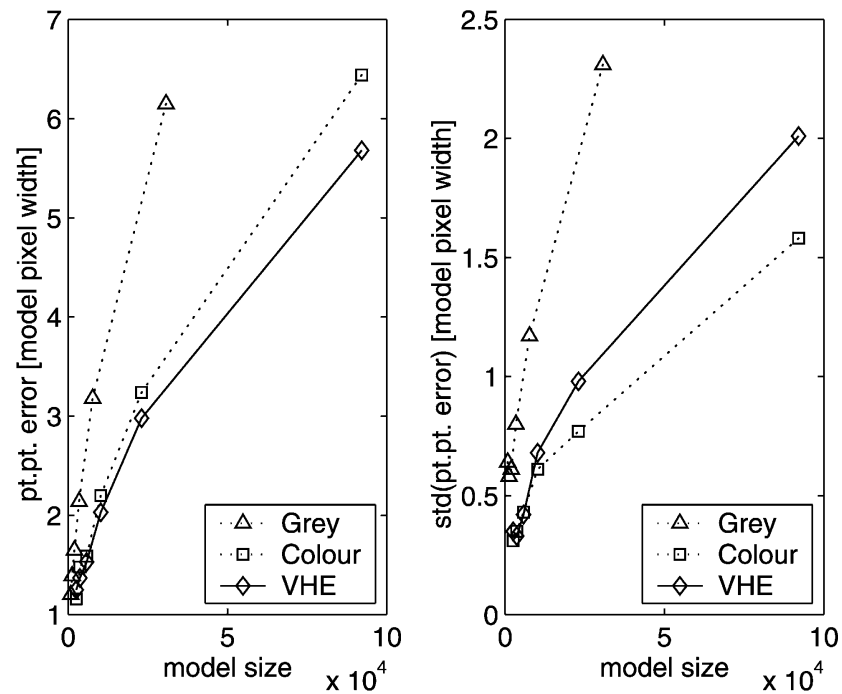

Fig. 5. AAM pt.-pt. accuracy measured as image pixel size vs model size (left) and std. of image pixel size vs model size.
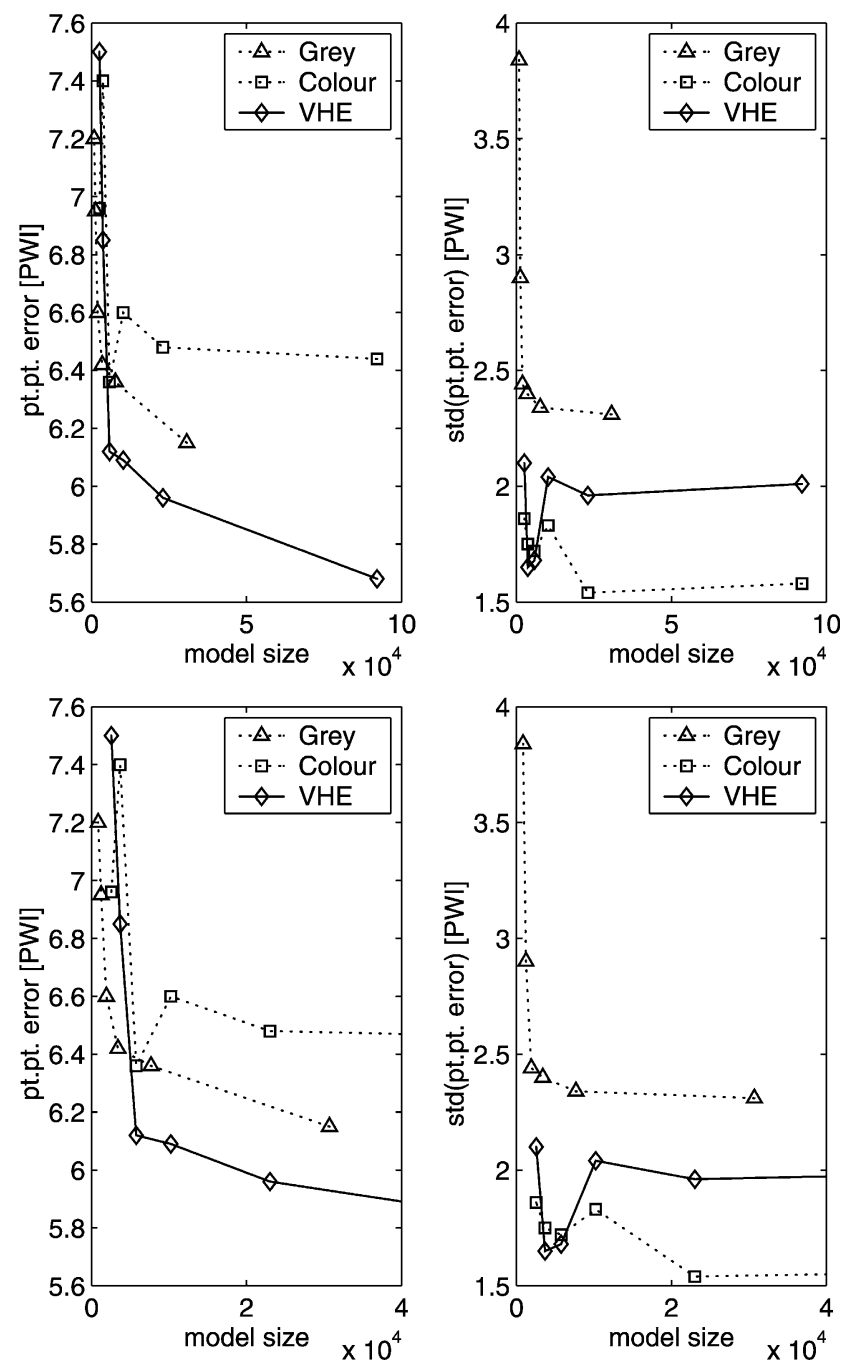

Fig. 6. AAM pt.-pt. accuracy measured as PWI vs model size (left) and std. of PWI vs model size in full (top) and zoomed (bottom) view. 
is seen that the VHE representation performs best for models larger than $\sim 5000$ texture samples.

If the choice should only regard the resolution of greyscale AAMs Fig. 6 (bottom) shows that the rate of improvement in PWI accuracy is far smaller for models with more than $\sim 3000$ texture samples.

Remarkably, the colour AAM had the lowest over-all PWI accuracy and unclear trends in both PWI mean and PWI standard deviation plots.

\section{Discussion}

Experiments have shown that a simple pre-processing of input images can increase segmentation accuracy on a limited set of facial images. The VHE representation outperforms conventional grey-scale AAMs and colour AAMs in cases with diffuse and partial directional illumination. The gain is obtained with negligible computational costs compared to colour AAMs but at the cost of a three times larger texture model compared to grey-scale AAMs.

Though stretched for display reasons, Fig. 3 indicates areas where the hue is ill-defined, e.g. at the eyes where the saturation is near zero. Further, in this discrete 24 bit RGB setting, pixels near zero intensity also results in ill-defined hue angles. These areas could be learned from the training set and subsequently down-weighted. This would lead to better models.

Circumventing the need for two texture bands to represent the cyclic hue as shown may be too primitive. Colour ambiguity is introduced at all colour angels $\pm \theta$ measured from the point of collapse (in this case pure red at angle 0). However, in the presented case the loss in colour specificity is more than compensated by the gain from the over-all decrease in lighting sensitivity. This may not always be the case. For human faces though, hue is concentrated around the angle $0 / 360^{\circ}$ [22]. For applications with limited and approximate unimodal distribution of hue-other than faces-the circular mean should be estimated [23] to ensure a proper representation. For multimodal cases two texture bands should be used.

Concatenating all bands with a subsequent common linear normalisation as done in AAMs seem less optimal. In the VHE case the three subbands all have substantially different statistics suggesting that bands should be normalised separately using possibly non-linear means of normalisation. This was earlier done on ultrasound images [10] with great success. In an initial stage this was applied to the edge band with limited success. The error increased due to the emphasis that was put on the noisy low to medium intensities, i.e. areas where the gradient are ill-defined. This could possibly be solved by a non-linear edge weighting scheme as suggested in Ref. [14] or by using a more elaborate regularisation prior to the gradient estimation, e.g. the anisotropic Perona-Malik diffusion scheme [24] or similar.

Finally, instead of patching the problem of non-Gaussian sources a more graceful solution would be to address the core of the problem. Namely, that PCA is based on assumptions of normally distributed variables. Independent component analysis (ICA) [25] could prove to be a good replacement of PCA.

\section{Conclusions}

Given the presence of colour information in a face segmentation task we have experienced the presented VHE representation to be an appealing alternative to model raw RGB intensities, in particular when dealing with change in lighting conditions. Using diffuse and partial directional lighting and at multiple scales, the VHE representation yielded higher accuracy than the conventional grey-scale and colour AAM. Only for very small models, grey-scale AAMs were more accurate.

From the current experiments, the VHE representation should be preferred over the colour representation. Further, compared to the grey-scale representation, the VHE should also be preferred if the required extra memory and computational power are available. For applications other than face segmentation, we have suggested modifications needed to utilise this intensity, hue and edge representation.

We have sought to promote the idea of modelling derived features combined with intensity information. Results have shown that with subtle changes to a traditional grey-scale AAM framework and simple domain specific pre-processing a considerable increase in accuracy can be obtained. We anticipate that this also holds for other domains.

\section{Acknowledgements}

The following people are gratefully acknowledged for their help in this work. The face database was built by Michael Moesby Nordstrøm, Mads Larsen and Janusz Sierakowski. Dmitry Karasik made the multi-band extension of the AAM-API.

\section{References}

[1] S. Sclaroff, J. Isidoro, Active blobs, Proceedings of the International Conference on Computer Vision, 1998, pp. 1146-1153.

[2] J. Isidoro, S. Sclaroff, Active voodoo dolls: a vision based input device for nonrigid control, Proceedings of Computer Animation'98, IEEE Computational Society, 1998, pp. 137-143.

[3] T. Vetter, Learning novel views to a single face image, Proceedings of the Second International Conference on Automatic Face and Gesture Recognition, 1996, pp. 22-27. 
[4] M. Jones, T. Poggio, Multidimensional morphable models: a framework for representing and matching object classes, International Journal of Computer Vision 29 (2) (1998) 107-131.

[5] G.J. Edwards, T.F. Cootes, C.J. Taylor, Face recognition using active appearance models, Proceedings of the Fifth European Conference on Computer Vision, ECCV'98, vol. 2, Springer, New York, 1998, pp. 581-595.

[6] T.F. Cootes, C.J. Taylor, Statistical models of appearance for medical image analysis and computer vision, Proceedings of SPIE Medical Imaging, vol. 1, SPIE, 2001, pp. 236-248.

[7] S. Mitchell, B. Lelieveldt, R. Geest, H. Bosch, J. Reiber, M Sonka, Time continuous segmentation of cardiac $\mathrm{mr}$ image sequences using active appearance motion models, Medical Imaging 2001: Image Processing, San Diego CA, SPIE, vol. 1, SPIE, 2001, pp. 249-256.

[8] S. Mitchell, B. Lelieveldt, R. van der Geest, H. Bosch, J. Reiver, M. Sonka, Multistage hybrid active appearance model matching: segmentation of left and right ventricles in cardiac $\mathrm{mr}$ images, IEEE Transactions on Medical Imaging 20 (5) (2001) 415-423.

[9] M.B. Stegmann, R. Fisker, B.K. Ersbøll, Extending and applying active appearance models for automated, high precision segmentation in different image modalities, Proceedings of the 12th Scandinavian Conference on Image Analysis-SCIA, vol. 1, 2001, pp. 90-97.

[10] H. Bosch, S. Mitchell, B. Lelieveldt, F. Nijland, O. Kamp, M. Sonka, J. Reiber, Active appearance-motion models for endocardial contour detection in time sequences of echocardiograms, Proceedings of SPIE-The International Society for Optical Engineering 4322 (1) (2001) 257-268

[11] H.H. Thodberg, Hands-on experience with active appearance models, Medical Imaging 2002: Image Processing, San Diego CA, SPIE, SPIE, 2002

[12] G.J. Edwards, C.J. Taylor, T.F. Cootes, Interpreting face images using active appearance models, Proceedings of the Third IEEE International Conference on Automatic Face and Gesture Recognition, IEEE Computational Society, 1998, pp. $300-305$.
[13] T.F. Cootes, G.J. Edwards, C.J. Taylor, Active appearance models, Proceedings of the European Conference on Computer Vision, vol. 2, Springer, New York, 1998, pp. 484-498.

[14] T.F. Cootes, C.J. Taylor, On representing edge structure for model matching, Proceedings of IEEE Computer Vision and Pattern Recognition-CVPR, vol. 1, IEEE, 2001, pp. 1114-1119.

[15] M.A. Turk, A.P. Pentland, Face recognition using eigenfaces, Proceedings of IEEE Computational Society Conference on CVPR, IEEE Computational Society Press, Silver Spring, MD, 1991, pp. 586-591.

[16] J.C. Gower, Generalized procrustes analysis, Psychometrika 40 (1975) 33-50.

[17] F.L. Bookstein, Principal warps: thin-plate splines and the decomposition of deformations, IEEE Transactions on Pattern Analysis and Machine Intelligence 11 (6) (1989) 567-585.

[18] T.F. Cootes, G.J. Edwards, C.J. Taylor, Active appearance models, IEEE Transactions on Pattern Recognition and Machine Intelligence 23 (6) (2001) 681-685.

[19] T.F. Cootes, C.J. Taylor, Statistical models of appearance for computer vision, Technical Report, October 2001, University of Manchester, http://www.isbe.man.ac.uk/ bim/, 2001.

[20] P. Switzer, A.A. Green, Min/max autocorrelation factors for multivariate spatial statistics, Technical Report 6, Stanford University, 1984, pp. 10.

[21] M.B. Stegmann, Object tracking using active appearance models, Proceedings of the 10th Danish Conference on Pattern Recognition and Image Analysis, Copenhagen, Denmark, vol. 1, DIKU, 2001, pp. 54-60.

[22] G. Bradski, Computer vision face tracking for use in a perceptual user interface, Intel Technology Journal, Intel Q2, 1998, http://www.intel. com/technology/itj/q21998.htm

[23] N.I. Fisher (Eds.), Statistical Analysis of Circular Data, Cambridge University Press, Cambridge, 1993.

[24] P. Perona, J. Malik, Scale space and edge detection using anisotropic diffusion, Proceedings of the IEEE Computer Society Workshop on Computer Vision (Cat. No. 87TH0210-5, 1987, pp. 16-22.

[25] P. Comon, Independent component analysis - a new concept?, Signal Processing 36 (1994) 287-314. 